\title{
Multicast Routing Protocol with Group-Level Congestion Prediction and Permanence under Constrained Energy Utilization for Mobile Ad Hoc Networks
}

\author{
K.Himabindu ${ }^{1}$, R.Sumalatha ${ }^{2}$ \\ ${ }^{I}$ (MTech. Student, Department of CS/IT, Srichaitanya College of Engineering, Karimnagar, AP, India) \\ ${ }^{2}$ (Senior Assistant Professor, Department of Cs/IT, Srichaitanya College of Engineering, Karimnagar, AP,
}

India)

\begin{abstract}
Here during this document A MAC layer stage overloading detection system has been foreseeable. The planned model aims to share out associate degree energy competent mechanism to calculate the degree of congestion at victim node with maximal accuracy. This block detection tool is incorporated with a sequence Crosslayered obstruction management Routing Topology. The projected model involves hard of congestion in 2 steps with effectual energy capable congestion detection and most favorable consumption of possessions. Packet loss in network routing is principally because of link failure and congestion. Most of the procurable congestion manages solutions don't have the flexibility to differentiate between packet loss owing to association collapse and packet loss unpaid to congestion. As a consequence these explanations aim towards exploiting against packet drop owing to link breaks down that could be a redundant endeavor and may turn out in defeat of resources. The opposite aspect within the majority of the simple to urge to resolve is that the consumption of power and resources to tell the apart jamming state, the amount of congestion and alert the premise node concerning congestion in routing path. Here during this document we tend to propose a cross lined model of congestion recognition and manage mechanism that contain power economical congestion discovery, Grouplevel Congestion Prediction (GCP) and Group-level Egress Permanence(GEP) algorithms that could work in sequence for congestion detection and manage model. This paper includes explorations and simulation results that demonstrate the increased store consumption, power potency in congestion discovery and congestion management is probable by the projected topology.
\end{abstract}

Keywords: quality of service, cross-layer protocols, multimedia communication, Low-power design, multicast routing

\section{Introduction}

The primary aim of a multicast topology is to transmit packets from a source node to the associates of a multicast group by means of a suitable quality of service (QoS) [2], [3]. QoS is the performance level of a service offered by the network, in general [1]. Specifically, QoS requires to maintain a high adequate packet delivery ratio (PDR), maintain the low packet drop and diminish the jitter in packet arrival times. Thus, the objective in QoS provisioning is to attain a further advanced network [1].

In fact, flooding, which is the easiest group message algorithm, is fine to attain elevated PDR subjected to the data transfer and(or) node density is not excessive in order that the network is not crammed. Conversely, flooding usually is not favored as a multicast routing topology because of its extreme make use of the obtainable bandwidth.

Therefore, the second aim of a multicast routing topology is to make use of the bandwidth competently, which is directly connected with the amount of repeated relay transmissions required to carry data packets to all associate nodes of a multicast group with an adequate PDR. The third aim of a multicast topology is to diminish the energy indulgence of the network.

Even though balancing the process of a wireless communication system by incorporating cross-layer design is a tempting choice, quite a lot of research work attempted to claim that such a cross-layer proposal is not the best option in the elongated usage since it may give up modularity and can lead to accidental cross-layer communications [6], [19]. Yet, by severely sticking on to a usual layering model, we may fail to notice on performance developments that be able to obtain in the course of the utilization of the less constrained crosslayer design gap [19], [20], [21]. Consequently, in this paper, we put forward a multicasting model based on cross-layer design while working out the extreme concern to ignore accidental cross layer communications.

Even though there are a lot of multicasting topologies for MANETs [4], [5], [7], [8], [9], it is clear from the most frequently cited literature, no topology is addressing together of QoS, spatial reuse efficiency, and overall energy indulgence. In this regard, here we propose a Multicast ad hoc routing that controls the congestion through a cross layered model that is based on the hierarchical egress normalizing approach. 
The paper is organized in various sections as follows: In section 2 we discuss the developments in multicast routing protocols for MANETs. In Section 3 we discuss the proposed congestion control under constrained energy utilization for multicast routing. In section 4 we describe the proposed model with requisite notations. In section 5 we give the model for Group level Congestion Prediction, and in section 6 we present the Group level Egress Permanence algorithm. Finally, we conclude with simulations and discussion of results in section 7.

\section{Related Work}

There are numerous multicast routing protocols intended for mobile ad hoc networks [8], [13], [15], [24], [29], and they can be classified into two extensive groups [13]: tree-based and mesh-based topologies. Tree-based topologies generate trees starting at the source node and coming to an end at multicast group associates by means of an intention of diminishing a cost utility. A multicast topology for ad hoc wireless networks (AMRIS) [4] builds a common distributed tree fixed at one of the nodes, with IDs rising as they give off from the starting node. Intra route renovation is made potential owed to this feature of the IDs, thus, reducing the route finding time and as well as controlling route restoring operating cost to the immediacy of the link breakdown.

Mesh-based multicasting is best suited to extremely dynamic topologies, merely owing to the redundancy related to this approach [5], [7]. In mesh-based approaches, there's over one path between the supply and also the multicast cluster members (i.e., a redundant multicast tree). One such mesh-based multicast topology, On-Demand Multicast Routing Protocol (ODMRP) [5], relies on periodic flooding of the network by the supply node through management packets to make a multicast mesh. This basic operation is employed each to make the initial multicast forwarding state and to take care of the mesh just in case of node quality and alternative network dynamics.

In ODMRP, an energetic supply sporadically floods the network with be a part of query management packets. once a node receives a be a query packet, it marks the primary node it receives the packet from as the upstream node it rebroadcasts the query packet. once a multicast cluster member receives a be a part of question packet, it replies back with a be a part of REPLY packet, that is forwarded back to the supply node via traversing the reverse path. every upstream node sets a forwarding flag for the multicast group indicated within the packet header and becomes a member of the multicast mesh. The forwarding state expires after a planned time.

A wealth of studies on energy-efficient multicasting in ad hoc networks can be found in [8], [29]. In [9], the matter of building a minimum energy multicast tree (i.e., specified the entire transmission power consumption within the multicast tree is minimized) for a given set of multicast nodes inside an ad hoc network is investigated. Since the matter is NP-complete, an approximation algorithmic program with demonstrable approximation assurance is proposed. An energy efficient multicasting algorithmic program for wireless networks with fastened transmit power nodes is given in [25]. In [26], a passive clusturing algorithmic program, that considers stability as well as residual energy of neighbouring nodes when choosing cluster heads and gateways, is proposed. This algorithmic considerably reduces routing-related control overhead. This model focuses on multicast services in wireless LANs. In [27], an approximation algorithmic with definite approximation ratios for decreasing the total energy consumption of treebased all-to-all multicasting in wireless ad hoc networks is devised. In [28], a self-managing, energy-efficient multicast routing suite based on the selfstabilization paradigm is proposed. However, the energy dissipation models employed in these studies include transmit and/or receive energy dissipation terms, whereas this could be a good approximation for some radios, there are other energy dissipation modes (i.e., idle, carrier sense, and sleep modes) for several current radios [14]. Hence, all the energy dissipation sources should be considered when constructing a multicasting protocol.

\section{Congestion Control Under Constrained Energy Resource Utilization For Multicast Routing}

\subsection{Congestion prediction under constrained energy utilization mechanism}

The aim of the proposed congestion detection machine is to capture the degree of overcrowding at relay hop level node with top precision. In the present model, the detection mechanisms are decoupled from different activities of the mackintosh stratum like link consistency analysis and protect size analysis. The acknowledgment model extended to note the congestion at traffic level, which depends on the number of congestion levels at the relay hop level node.

\subsubsection{Measuring degree of jamming of the Relay hop level node:}

Unlike traditional networks, nodes within the ad hoc set-up exhibit a higher degree of non uniformity in terms of each hardware and package configurations. The non uniformity of the die hop nodes will reflect as numerous radio vary, limit retransmission add up, and barrier capability. Thus the degree of channel loading, 
packet drop rate, and therefore the quantity of buffer utilization at the relay hop height node may be a least combination to search out the degree of obstruction. The usage of those 3 determined values supports to decouple the obstructive live development from further Mac layer behavior.

The level of channel load, packet drop rate and level of buffer method collectively offer a scope to imagine the overcrowding, causing unfortunate magnitude relation between crash and retransmission count up. Once retransmissions peculiar to crash rate are significantly low, then resolution delay of die hop node can augment proportionately, that escort to obstructive and replicate as blockage attributable to safeguard run over.

\subsubsection{Measuring degree of congestion at path level traffic}

The scale of congestion at each neighbor hop helps to form the extent of obstruction at path level transfer from supply to target node. Each relay hop level node takes delivery of the degree of overcrowding from its entry point. Since the explanation node, that is the last node of the direction-finding path doesn't reply, the target node initiates to assess the quantity of overcrowding at path level traffic. The periodic updates of overcrowding position at every corresponding hop height node to its successor in direction-finding path are notably energy overwhelming activity. Hence to avoid wasting the energy, the interference update approach decide 2 restricted actions as follows:

1. Amount of blocking $d_{c}\left(h_{i}\right)$ at go by on the jump phase node $h_{i}$ will be sent to its heir $h_{i+1}$ if the ' $d_{c}\left(h_{i}\right)$ ' is larger than the node height obstacle threshold $d_{c}(\tau)$. So the power protect due to restrictive transmission.

2. If the extent of jamming at the corridor altitude interchange $d_{c}(r p)$ that traditional by nodule $h_{i}$ from its entrance inventor $h_{i-1}$ is lesser than $d_{c}\left(h_{i}\right)$ then it inform the $d_{c}(r p)$ else it remnants same, hence force conserve due to prevention of $d_{c}(r p)$ inform.

\section{Congestion Prediction And Permanence Under Constrained Energy Utilization Mechanism}

The packet dropping often occurs in Manet's. The reason for this packet dropping is as below

Transmission Link failure.

- Inferred transmission due to flooded input that leads Ingress receiving strength to be low. This can be claimed as packet dropping due to jamming at routing.

Here we divide the network in to groups, for each group, group leader will be selected and then the level of congestion will be calculated in two stages as below

- The level of congestion within the Multicast-group stage.

- The level of congestion in various Multicast-group stages.

With this the resource level cost may be reduced and the energy required may be regulated.

Multicast- group
$I C E A$
$I E R A$
$E R A$
$D P G$
$E I L$
$L F L$
$I R S$
$I R S_{p}$
$I R S_{e}$
$R P$
$d t_{n}$
$N$
$Z n_{i}$
$z h_{i}$
$z h_{i}^{\prime}$
$Z_{c}$
$Z_{p}$
$Z_{f}$
$Z_{i}$

\subsection{Notations used in the proposed model}

A physical area, which is the division of favored mobile ad hoc association Intra level congestion Evaluation Algorithm

Intra level Egress Regularization Algorithm

Egress Regularization Algorithm

Distance Power Gradient

Ingress inferred Loss

Link Failure Loss

Ingress receives strength

Present Ingress receives strength

Expected Ingress Receiving Strength

Routing path

Delay time at node $n$

Number of nodes in entire network

Number of nodes in a Multicast-group $i$

Multicast-group head of the $i^{\text {th }}$ Multicast-group

Reserved Multicast-group head of the $i^{\text {th }}$ Multicast-group

Current Multicast-group in the hierarchy

Preceding Multicast-group to the current Multicast-group $Z_{c}$ in the hierarchy

Following Multicast-group to the current Multicast-group $Z_{c}$ in hierarchy

$i^{\text {th }}$ Multicast-group in the routing path 
Multicast Routing Protocol With Group-Level Congestion Predection And Permanence Under

$n_{z} \quad$ Multicast-group of the node $n$

$\zeta_{Z} \quad$ MaximumMulticast-group level Transmission Load

$\zeta_{n}$

Maximum Node level Transmission Load

$\zeta_{T}$

$\zeta_{t}$

Predefined Threshold that represents gap between two transmissions at one hop

level

Actual gap between last two transmissions

$\zeta_{\text {et }}$

$I R S_{\zeta_{T}}$

Elapsed time since previous transmission at one hop level

$\delta^{\prime}$

Maximum average ingress receiving strength observed for predefined gap $\zeta_{T}$

$I R S_{c e}$

Standard sloping threshold of the receiving strength

$I R S_{r}$

Estimated maximum ingress receiving strength at current interval

$I R S_{c r}$

ingress receiving strength ratio

Current input receiving strength ratio

$B T_{n}$

Buffer time at node $n$

$z$ dil $_{i}$

Multicast-group level degree of ingress load, here $i$ is a group id.

$\operatorname{ndil}_{k}$

Node level degree of ingress load, here $k$ is the node id of the $\operatorname{group}_{i}$

\subsection{Network and Node activities under projected protocol}

The network is to be divided into Multicast-groups

For every Multicast-group $i$ where $\mathrm{i}=1,2, \ldots|\mathrm{Z}|(|\mathrm{Z}|$ is total number of groups)

Select Multicast-group-head for each Multicast-group $i$

Find maximum transmission load $\zeta_{n}$ for every Multicast-group $i$

By using $\zeta_{n}$ of each Multicast-group measure the maximum transmission load for the whole network.

\subsection{Splitting the network into Multicast-groups}

We choose to the approach described by Mohammad M. Qabajeh et al [8]. The ad hoc network region is partitioned into equal size groups and it must be known to all the nodes participating in the network. The hexagon is chosen as the group shape as it can cover a two-dimensional region completely without any overlap and also it enables communication with more neighbors. The transmission range of a node is denoted as $\mathrm{R}$ and the side length of hexagon as $\mathrm{L}$. The connection between $\mathrm{R}$ and $\mathrm{L}$ is set as $\mathrm{L}=\mathrm{R} / 2$ by which each two nodes inside the group can communicate with each other directly.

Every Multicast-group has a Multicast-group identity (zid), Multicast-group head $(z h)$ and Multicastgroup head reserve $\left(z h^{\prime}\right)$. The $z h$ node maintains information about all the nodes in a Multicast-group with their positions and IDs. Also, it is responsible to maintain information about the group heads of the neighboring Multicast-groups as shown in the figure 1. The duty of CLB node is to keep a copy of the data stored at the $z h$ in order not to be lost when the $z h$ node is off or moving the Multicast-group. By knowing the coordinates of a node position,nodes can perform our self-mapping algorithm of their physical locations onto the present Multicast-group and calculate its zid easily. Figure 1 shows the general summary of the network architecture.

\subsection{Selecting Multicast-group-Heads}

A Multicast-group-Head selection occurs basis on the following terms:

I. Node position: A node in the location $p$ that is close to the center of the multicast group is most favorable to act as Multicast-group head.

II. Energy level: A node with maximum energy level e is preferable to act as Multicast-group head.

III. Computational capability: The node with high computational ability c is optimal to act as a Multicastgroup Head.

IV. Mobility: The node with less mobility $m$ is acceptable to act as a zone head.

Each node of the Multicast-group broadcasts its $(p, e, c, m)$. The node which has all the above $(p, e, c, m)$ metrics is declared itself as group head $z h$. The next optimal node in the series becomes the reserve Multicastgroup head $z h^{\prime}$. 


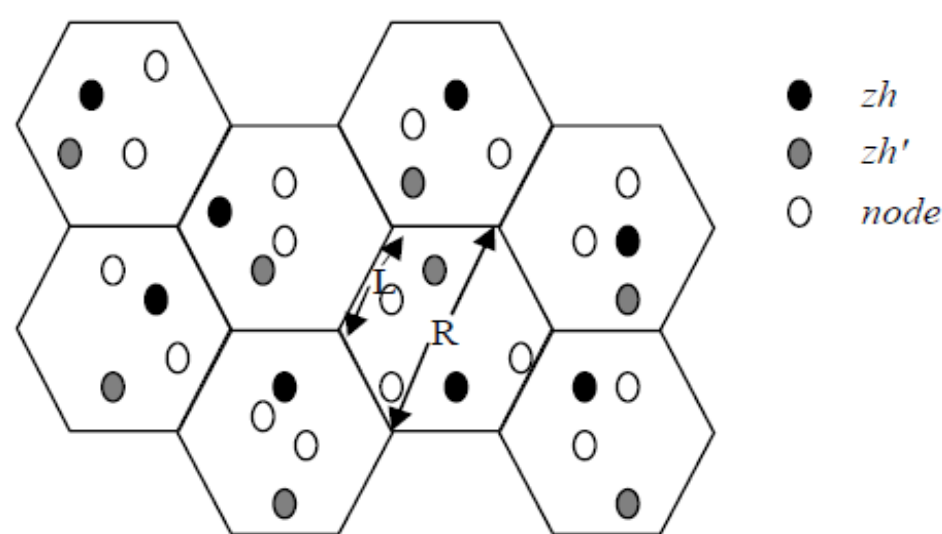

Figure 1[8]: Architecture of the Multicast-group partitions in network.

\subsection{Information sharing at intra Multicast-group level (between multicast-group head and node)}

Each node $\mathrm{n}$ that fit in to Multicast-group $\mathrm{Z}$ verifies the incoming load and shares degree of ingress load $d_{i l} l_{n}$ with Multicast-group head. Once $n d i l_{k}$ received from each node $k$ of the Multicast-group $i$, the Multicast-group head $z h$ calculates the degree of ingress load at Multicast-group level $z d i l_{i}$.

$$
z d i_{z_{i}}=\frac{\sum_{k=1}^{z n_{i}} n d i l_{k}}{z n_{i}}
$$

\section{Group-Level Congestion Prediction (GCP) Algorithm}

Multicast-group level Congestion Predicition algorithm abbreviated as GCP is presented in this section. GCP is the finest algorithm that helps in locating the packet drop under congestion. This is evaluated by Mac layer and then it alerts the network layer.

\section{Fig2: GCP for estimating status of congestion caused due to packet drop}

\begin{tabular}{|c|c|}
\hline $\begin{array}{l}\text { At an event of ingress receiving by node } i \text { : } \\
\text { Updating Ingress receiving strength: } \\
\text { if } \zeta_{t}<\zeta_{T} \text { then } \\
\delta^{\prime}=\frac{1}{2}\left(\frac{I R S_{c r}-I R S_{\zeta_{T}}}{\zeta_{t}}\right)+\frac{1}{2} \delta^{\prime} \\
I R S_{\zeta_{T}}=I R S_{c r}\left(\frac{\zeta_{t}}{\zeta_{T}}\right)+I R S_{\zeta_{T}}\left(\frac{\zeta_{T}-\zeta_{t}}{\zeta_{T}}\right) \\
\text { end if } \\
\text { if } \zeta_{t} \geq \zeta_{T} \text { then } \\
\delta^{\prime}=\left(\frac{I R S_{c r}-I R S_{\zeta_{T}}}{\zeta_{t}}\right) \\
I R S_{\zeta_{T}}=I R S_{c r} \\
\text { end if }\end{array}$ & $\begin{array}{l}\text { Detecting packet drop at the Mac layer level } \\
\text { IRS } \\
\text { if }=I R S_{\zeta_{T}}+\delta^{\prime} \zeta_{e t} \\
\text { IRS } \\
\text { Mac Alert link failure } \\
\text { Else } \\
\text { Mac Alert Congestion }\end{array}$ \\
\hline
\end{tabular}

\section{Group-Level Egress Permanence (GEP) Algorithm}

This event occurs if Mac-layer alert indicates the congestion situation. Once the routing protocol [13] gets an alert from the Mac layer about the congestion at a node $i$, it alerts the adjacent node that is the source node $s$ for conflict node $i$. Hence $s$ calculates it's $d i l_{s}$ by comparing with $z d i l$ of $Z_{c}$ (Multicast-group of the node s). If $d i l_{s}$ is greater than $z d i l_{z_{c}}$ and difference between $d i l_{s}$ and $z d i l_{s_{2}}$ is greater than equal to egress threshold $\varepsilon$ then node $s$ regularize the outflow by increaing its buffering time $B T_{s}$ such that $n d i l_{s} \geq z d i l_{s_{z}}+\varepsilon_{s_{z}}$.

Here $\varepsilon$ can evaluated with the following equation 


$$
\varepsilon_{j}=\frac{\sum_{k=1}^{z n_{j}} z d i l_{j}-d i l_{k}}{z n_{j}}
$$

In case if the node $s$ not able to regularize its outflow such that conflict node i prevents from congestion then it alerts the $z h_{s_{z}}$ (Multicast-group-head of the $Z_{c}, s \in Z_{c}$ ). In the series of that event $z h_{z_{c}}$ alert all the nodes in the group. Thus the nodes those are in upstream side of the source node $s$ in routing way attempt to regularize their egress load using the above method in this section. Then all nodes update there ndil and send to Multicast-group-head $z h_{z_{c}}$, then Multicast-group-head $z h_{z_{c}}$ calculates $z$ dil and verifies reliability of the $z$ dil by comparing with $d i l . \quad z d i l_{Z_{c}} \geq d i l+\bar{\varepsilon}$ concludes that congestion at conflict node is controlled by egress regularization at current Multicast-group level. If $z d i l_{z_{c}}<d i l+\bar{\varepsilon}$ then congestion evaluation algorithm will be initiated at $Z_{p}$, which is immediate upstream Multicast-group to $Z_{c}$ in hierarchy. In this procedure Multicastgroup head of the $Z_{c}$ initially alerts the Multicast-group head of the counterpart $Z_{p}$ then $z h_{z_{p}}$ alerts all nodes that belong to $Z_{p}$, which are part of the route path. The above process of egress regularization at the multicast-group level can be referred as GEP (Group-level Egress Permanence) algorithm. Therefore the nodes belong to $Z_{p}$ attempt to regularize their egress load by using GEP and alert Multicast-group head about their updated degree of ingress load $n$ dil. Then ' $z h_{z_{p}}$ ' calculates ' $z d i l_{z_{p}}$ ' and verifies whether $z d i l_{Z_{p}} \geq d i l+\bar{\varepsilon}$ true or false.True indicates that the jamming at the conflict node has been reduced or removed because of the egress load regularization at Multicast-group $Z_{p}$, if false then Multicast-group head of the $Z_{p}$ alerts all other Multicastgroup heads using a broadcasting [12] approach about the congestion at immediate zone in downstream of the hierarchy . Hence all Multicast-groups in the upstream side of the $Z_{p}$ apply GEP and the Multicast-groups in the downstream side of the $Z_{p}$ updates their $z$ dil . Then all Multicast-groups send their $z$ dil to source Multicastgroup. Hence the base Multicast-group evaluates the dil .Basing on the dil , source node regularizes its egress load.

\section{Fig 3: Group-level Egress Permanence(GEP)}

\begin{tabular}{|l|l|}
\hline The notations used in Algorithm: \\
\hline$i$ & Node that had been affected by emptiness. \\
\hline$S$ & Source node of $i$. \\
\hline$Z_{c}$ & Present Multicast-group where $i, s \in Z_{c}$ \\
\hline$Z_{p}$ & Immediate Multicast-group to $Z_{c}$ in upstream side of the pecking order. \\
\hline$\left\{n_{u_{i}}\right\}_{Z_{c}}, i=1,2, \ldots, k$ & All upstream nodes to $s$. \\
\hline$\left\{n_{d_{i}}\right\}_{Z_{c}}, i=1,2, \ldots, k$ & All downstream nodes to $s$. \\
\hline$Z_{S} \cup\left\{Z_{u_{i}}\right\}, i=1,2, \ldots, k$ & $\begin{array}{l}\text { Set of upstream Multicast-groups to } Z_{p} \text { in the routing path, here } Z_{S} \text { is a } \\
\text { Multicast - group that contains the source node of the course-plotting path. }\end{array}$ \\
\hline$\left\{Z_{d_{i}}\right\} \cup Z_{T}, i=1,2, \ldots, m$ & $\begin{array}{l}\text { Set of downstream Multicast-groups to } Z_{p} \text { in routing path, here } Z_{T} \text { is a } \\
\text { Multicast - group that have target node of the routing path. }\end{array}$ \\
\hline$\varepsilon$ & Multicast - group level way out threshold. \\
\hline $\bar{\varepsilon}$ & System level way out threshold. \\
\hline
\end{tabular}

\begin{tabular}{l}
\hline Algorithm \\
Mac layer alerts about the jamming at the node of the multicast-group $Z_{c}$ to routing protocol, hence the \\
next steps performed in order \\
$\varepsilon_{Z_{C}}=\frac{\sum_{k=1}^{z n_{Z_{C}}} z d i l_{Z_{C}}-d i l_{k}}{z n_{Z_{C}}}$ \\
\hline
\end{tabular}


At node $s$ the following sequence is carried out

if ndil $_{s}>z d i l_{Z_{c}}$ and $n$ dil $_{s}-z$ dil $_{Z_{c}} \geq \varepsilon_{Z_{c}}$ then

$B T_{s}=B T_{s}+b t$

Note: Value of buffer threshold bt should be certain such that dil $l_{S} \geq z d i l_{Z_{C}}+\varepsilon_{Z_{C}}$

return

end if

$s$ sends alert to $z h_{Z_{c}}$ about conflict node $i$.

$z h_{Z_{c}}$ alerts all nodes that belong to Multicast-group $Z_{c}$

$\left\{n_{u_{i}}\right\}_{Z_{c}}, i=1,2, \ldots, k$ updates their $n d i l$ by apply CON recursively and alerts $z h_{Z_{c}}$

$\left\{n_{d_{i}}\right\}_{Z_{c}}, i=1,2, \ldots, k$ events their $n d i l$ and alerts $z h_{Z_{c}}$

$z h_{Z_{c}}$ actions $z d i l$ as fallows

$z d i z_{z_{c}}=\frac{\sum_{k=1}^{z n_{Z}} n d i l_{k}}{z n_{Z_{c}}}$

if $z$ dil $_{Z_{c}}>$ dil and $z d i l_{Z_{c}}-$ dil $\geq \bar{\varepsilon}$ then

Alert: blocking at contention node normalized at present Multicast-group $Z_{c}$ level.

Return.

Endif

$z h_{Z_{c}}$ alerts $z h_{Z_{p}}$

$z h_{Z_{p}}$ alerts all nodes that feel right to Multicast-group $Z_{p}$

For both nodes $n \in Z_{p}$ begin

$$
\begin{gathered}
\text { if } \operatorname{ndil}_{n}>z \mathrm{dil}_{Z_{p}} \text { and } n d i l_{n}-z d i l_{Z_{p}} \geq \varepsilon_{Z_{p}} \text { then } \\
\qquad B T_{n}=B T_{n}+b t
\end{gathered}
$$

Note: Value of hurdle threshold ${ }_{b t}$ should be determined such that dil $l_{n} \geq z d i l_{Z_{C}}+\varepsilon_{Z_{C}}$

endif

Find $d i l_{n}$ and send $d i l_{n}$ to $z h_{Z_{p}}$

End-of-for each

$z h_{Z_{p}}$ events $z d i l_{Z_{p}}$

if $z \operatorname{dil}_{Z_{p}}>\operatorname{dil}$ and $\left(z d i l_{Z_{p}}-\operatorname{dil}\right) \geq \bar{\varepsilon}$ begin

Alert: way out regularization at $Z_{p}$ leads to conquer congestion circumstances at disputation

Multicast-group.

Endif

$z h_{Z_{p}}$ alerts all Multicast-group heads in the complex about overcrowding disagreement Multicast-group.

For each Multicast-group $z$ in $Z_{S} \cup\left\{Z_{u_{i}}\right\}, i=1,2, \ldots, k$ begin

$z h_{z}$ alerts all nodes that belong to Multicast-group $z$

For each node $n \in z$ begin

$$
\text { If } n d i l_{n}>z d i l_{z} \text { and } n d i l_{n}-z d i l_{z} \geq \varepsilon_{z} \text { begin }
$$

$$
B T_{n}=B T_{n}+b t
$$

Note: Value of buffering threshold bt should be assumed such that

$$
\operatorname{dil}_{n} \geq z d i l_{Z}+\varepsilon_{Z}
$$

endif

Find $\operatorname{dil}_{n}$ and send dil $l_{n}$ to $z h_{z}$

End-of-for each

$z h_{z}$ measures $z d i l_{z}$ and advances towards source Multicast-group.

End-of-for each 
For each Multicast-group $z$ in $\left\{Z_{d_{i}}\right\} \cup Z_{T}, i=1,2, \ldots, m$ begin

For each node $n$ fit in to Multicast-group $z$ begin

Concludes $n d i l_{n}$ and sends to $z h_{z}$

End-of-for each

$z h_{z}$ procedures $z d i l_{z}$ as $z d i l_{Z}=\frac{\sum_{k=1}^{z n_{Z}} n d i l_{k}}{z n_{Z}}$

$z h_{z}$ sends $z d i l_{z}$ to source Multicast-group through broadcasting [12]

End-of-for each

$Z_{S}$ measures dil as $d i l=\frac{\sum_{i=1}^{|Z|} z d i l_{i}}{|Z|}$

Hence the source node $s$ regularizes its egress load to routing path.

\section{Simulations and results discussion}

In this section we discuss the end acquired from replication conducted using 'Madhoc simulator' [16] in this section. We evaluated concert using Madhoc with the following consideration:

\begin{tabular}{|l|l|}
\hline No of Hops: & 225 \\
\hline Estimated Hop distance & 300 meters \\
\hline Approximate total network & $1000 \mathrm{X} 1000$ meters \\
\hline $\begin{array}{l}\text { Quite accurate Multicast- } \\
\text { group Radios }\end{array}$ & $100 \mathrm{X} 100$ meters \\
\hline Physical channel bandwidth & $2 \mathrm{mbps}$ \\
\hline Mac Layer: & $\begin{array}{l}802.11 \mathrm{DCF} \text { with preference of } \\
\text { handshaking prier to data transferring }\end{array}$ \\
\hline Physical layer illustration & $802: 11 \mathrm{~B}$ \\
\hline Presentation Index & $\begin{array}{l}\text { way out regularization cost and end- } \\
\text { to-end throughput }\end{array}$ \\
\hline $\begin{array}{l}\text { Be very winning simulation } \\
\text { time }\end{array}$ & \begin{tabular}{l}
150 sec \\
\hline
\end{tabular}
\end{tabular}

The imitation is conducted on three routes opposing by the no of hops and length.

1. Short span path: A route with 15 hops

2. Middling span : A route with 40 hops

3. Max span: A route with 81 hops

The same load is agreeing to all the paths with a regular period of $10 \mathrm{sec}$. Consignment given in kilobytes is shown in fig 4. The fig 5 concludes the step up of GCP-GEP over jamming control procedure [31] in obstruction control cost. A. The overcrowding discovery cost evaluation between GCP-GEP and blocking control etiquette[31] is travelling around in fig 6 that elevate the vigor good group achieve under GCP-GEP. The process of capacity of congestion control and jamming uncovering cost is as follows:

Based on the reserve effortlessness of use, bandwidth and force, for character process a threshold value between 0 and 1 assign. In the instrument of overcrowding detection and direct the sum cost is calculated by summing the price threshold of every concerned event. In fig 5 the ruling between blockage costs observed for GCP-GEP and overcrowding and debate control mock-up [31] are shown.

$$
\cos t_{c h}=\sum_{e=1}^{E} c t_{e}
$$

Here $\cos t_{c h}$ is the price of an impasse scheming movement $c h, E$ is the total quantity of events built-in. $c t_{e}$ is the threshold cost of an event $e$. The illustration events are:

1." Alert to source node as of Mac layer" 
2. "Alert from node to Multicast-group head", "spread by Multicast-group skull to another Multicast-group head"

3. "Ingress ruling and egress regularization".

4. Alert about $d_{c}\left(h_{i}\right)$

5. Bring up to date $d_{c}(r p)$

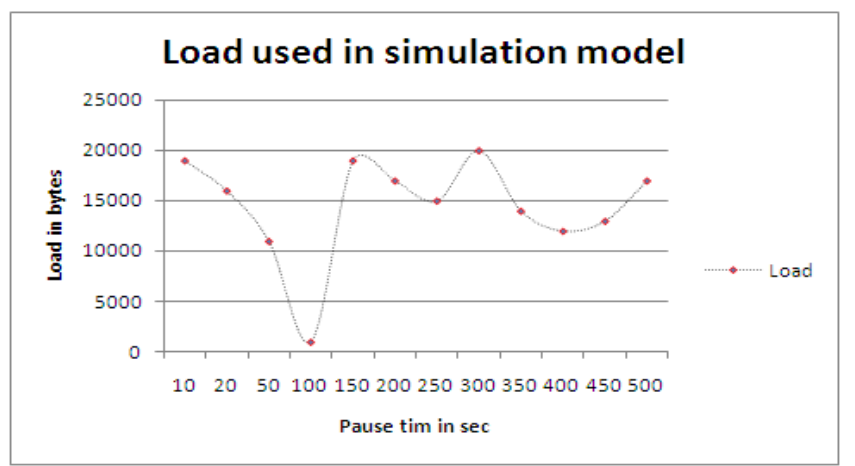

Fig 4: weight in bytes drive by foundation node of the direction-finding path (in regular interval of $10 \mathrm{sec}$ )

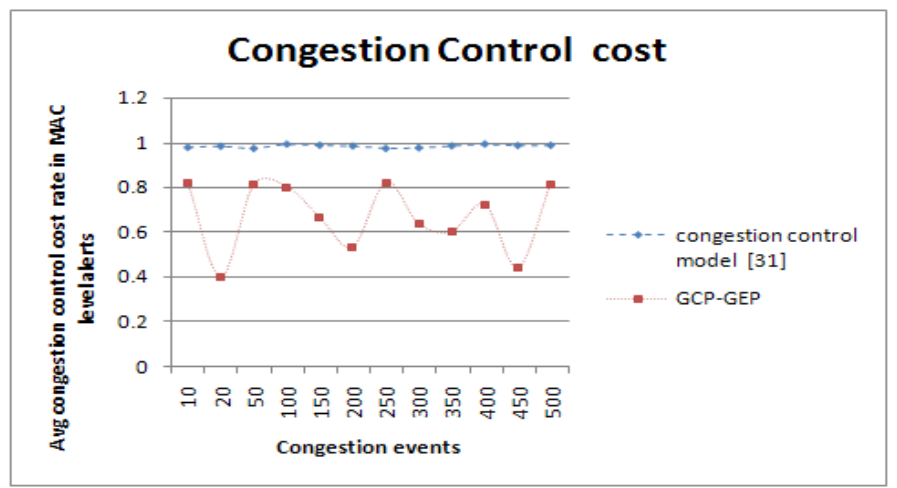

Fig 5: Congestion Control cost comparison chart

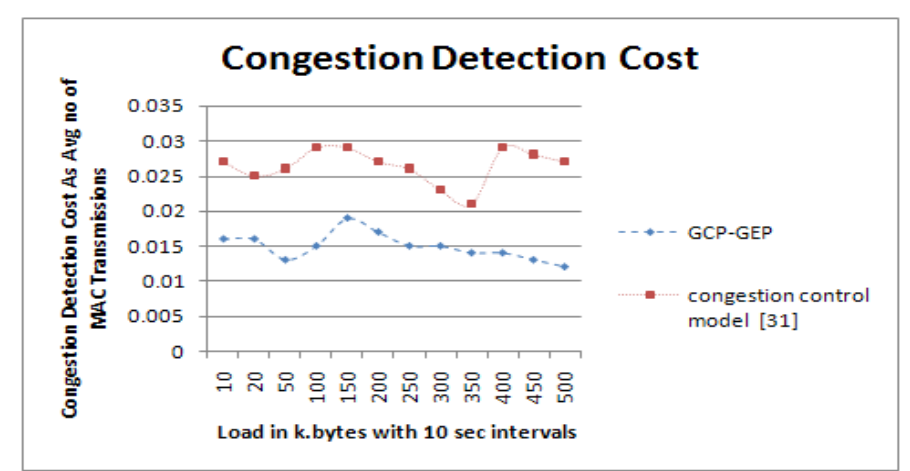

Fig 6: A line chart comparison of Congestion detection cost

\section{Conclusion}

This manuscript discussed proposed Multicast routing protocol with Group-level congestion Prediction and Permanence under constrained energy utilization for mobile Ad hoc Networks in short referred as GCPGEP. GCP-GEP derived a Cross layered congestion detection mechanism with energy effectiveness as primary criteria that included as congestion prediction mechanism. In this regard it opt to a hierarchical approach of congestion permanence of predicted. This hierarchical approach is efficient to perform under minimal resource utilization. The simulation results evident that the proposed model is significantly delivered better performance than other frequently cited energy efficient multicast routing models.

\section{References}

[1] C.S.R. Murthy and B.S. Manoj, Ad Hoc Wireless Networks: Architectures and Protocols. Prentice Hall, 2004.

[2] J. Janssen, D.D. Vleeschauwer, G.H. Petit, R. Windey, and J.M. Leroy, "Delay Bounds for Voice over IP Calls Transported over Satellite Access Links," Mobile Networks and Applications, vol. 7, pp. 79-89, 2002.

[3] B. Tavli and W. Heinzelman, Mobile Ad Hoc Networks: Energy- Efficient Real-Time Group Communications. Springer, 2006. 
[4] C.W. Wu and Y.C. Tay, "AMRIS: A Multicast Protocol for Ad Hoc Wireless Networks," Proc. IEEE Military Comm. Conf., vol. 1, pp. 25-29, 1999.

[5] S.J. Lee, W. Su, and M. Gerla, "On-Demand Multicast Routing Protocol in Multihop Wireless Mobile Networks," Mobile Networks and Applications, vol. 7, pp. 441-453, 2002.

[6] V. Kawadia and P.R. Kumar, “A Cautionary Perspective on Cross- Layer Design,” IEEE Wireless Comm., vol. 12, no. 1, pp. 3-11, Feb. 2005.

[7] J.G. Jetcheva and D.B. Johnson, “Adaptive Demand-Driven Multicast Routing in Multi-Hop Wireless Ad Hoc Networks," Proc. ACM Int'l Symp. Mobile Ad Hoc Networking and Computing, pp. 33-44, 2001.

[8] Mohammad M. Qabajeh, Aisha Hashim, Othman Khalifa \& Liana K. Qabajeh, 2010, "Geographical Multicast Quality of Service Routing Protocol for Mobile Ad-Hoc Networks", Journal of Engineering Letters, Vol. 18, No. 3, pp. 212-225.

[9] W. Liang, "Approximate Minimum-Energy Multicasting in Wireless Ad Hoc Networks," IEEE Trans. Mobile Computing, vol. 5, no. 4, pp. 377-387, Apr. 2006.

[10] B. Tavli and W. Heinzelman, "TRACE: Time Reservation Using Adaptive Control for Energy Efficiency,” IEEE J. Selected Areas Comm., vol. 21, no. 10, pp. 1506-1515, Dec. 2003.

[11] B. Tavli and W. Heinzelman, "MH-TRACE: Multi Hop Time Reservation Using Adaptive Control for Energy Efficiency," IEEE J. Selected Areas Comm., vol. 22, no. 5, pp. 942-953, June 2004.

[12] B. Tavli and W. Heinzelman, "Energy and Spatial Reuse Efficient Network Wide Real-Time Data Broadcasting in Mobile Ad Hoc Networking,” IEEE Trans. Mobile Computing, vol. 5, no. 10, pp. 1297-1312, Oct. 2006.

[13] H. Moustafa and H. Labiod, "A Performance Comparison of Multicast Routing Protocols in Ad Hoc Networks," Proc. IEEE Personal, Indoor and Mobile Radio Conf., pp. 497-501, 2003.

[14] Network Simulator (NS), http://www.isi.edu/nsnam/ns, 2010.

[15] S.J. Lee, W. Su, J. Hsu, M. Gerla, and R. Bagrodia, "A Performance Comparison of Ad Hoc Wireless Multicast Protocols," Proc. IEEE Conf. Computer Comm., pp. 565-574, 2000.

[16] W. Ye and J. Heidemann, "Medium Access Control in Wireless Sensor Networks," Technical Report ISI-TR-580, Information Sciences Inst., Univ. of Southern California, 2003.

[17] J.-P. Ebert, S. Aier, G. Kofahl, A. Becker, B. Burns, and A. Wolisz, "Measurement and Simulation of the Energy Consumption of an WLAN Interface," Telecomm. Network Group Technical Report TKN-02-010, Technical Univ. of Berlin, 2002.

[18] B. Tavli and W. Heinzelman, "MC-TRACE: Multicasting through Time Reservation Using Adaptive Control for Energy Efficiency," Proc. IEEE Military Comm. Conf., pp. 2076-2081, 2005.

[19] M. Conti, G. Maselli, G. Turi, and S. Giordano, "Cross-Layering in Mobile Ad Hoc Network Design," Computer, vol. 37, no. 2, pp. 48- 51, Feb. 2004.

[20] M. Van Der Schaar and S. Shankar, "Cross-Layer Wireless Multimedia Transmission: Challenges, Principles and New Paradigms," IEEE Wireless Comm., vol. 12, no. 4, pp. 50-58, Aug. 2005.

[21] V. Srivastava and M. Motani, "Cross-Layer Design: A Survey and the Road Ahead," IEEE Comm. Magazine, vol. 43, no. 12, pp. 112- 119, Dec. 2005

[22] M. Gerla, Y.-Z. Lee, J.-S. Park, and Y. Yi, "On Demand Multicast Routing with Unidirectional Links," Proc. IEEE Wireless Comm. and Networking Conf., pp. 2162-2167, 2005.

[23] T. Numanoglu, B. Tavli, and W. Heinzelman, "Energy Efficiency and Error Resilience in Coordinated and Non-Coordinated Medium Access Control Protocols,” Computer Comm., vol. 29, pp. 3493-3506, 2006.

[24] L. Junhai, Y. Danxia, X. Liu, and F. Mingyu, "A Survey of Multicast Routing Protocols for Mobile Ad-Hoc Networks," IEEE Comm. Surveys and Tutorials, vol. 11, no. 1, pp. 78-91, First Quarter 2009.

[25] C. Papageorgiou, P. Kokkinos, and E. Varvarigos, "Energy- Efficient Multicasting in Wireless Networks with Fixed Node Transmission Power," Proc. ACM Int'l Conf. Comm. and Mobile Computing, pp. 958-962, 2009.

[26] E. Astier, A. Hafid, and A. Benslimane, "Energy and Mobility Aware Clustering Technique for Multicast Routing Protocols in Wireless Ad Hoc Networks," Proc. IEEE Wireless Comm. and Networking Conf., pp. 1-6, 2009.

[27] W. Liang, R. Brent, Y. Xu, and Q. Wang, "Minimum-Energy Allto- All Multicasting in Wireless Ad Hoc Networks," IEEE Trans. Wireless Comm., vol. 8, no. 11, pp. 5490-5499, Nov. 2009.

[28] T. Mukherjee, G. Varsamopoulos, and S.K.S. Gupta, "Self Managing Energy-Efficient Multicast Support in MANETs under Endto-End Reliability Constraints," Computer Networks, vol. 53, pp. 1603-1627, 2009.

[29] S. Guo and O.W.W. Yang, "Energy-Aware Multicasting in Wireless Ad Hoc Networks: A Survey and Discussion," Computer Comm., vol. 30, pp. 2129-2148, 2007.

[30] B. Tavli and W. Heinzelman, "QoS and Energy Efficiency in Network-Wide Broadcasting: A MAC Layer Perspective," Computer Comm., vol. 30, pp. 3705-3720, 2007.

[31] Bulent Tavli and Wendi B. Heinzelman; Energy-Efficient Real-Time Multicast Routing in Mobile Ad Hoc Networks; IEEE TRANSACTIONS ON COMPUTERS, VOL. 60, NO. 5, MAY 2011 